\title{
EDITORIAL
}

\section{Bacterial infection and chronic obstructive pulmonary disease}

\author{
R. Wilson
}

Antibiotics are commonly prescribed empirically to patients presenting with an acute exacerbation of chronic obstructive pulmonary disease (COPD) to treat presumed bacterial infection. The rise in bacterial resistance to antibiotics has focused our attention on the benefit of this practice, and more fundamentally the importance of bacterial infection in COPD and its role in stimulating the bronchial inflammation that is the hallmark of this condition $[1,2]$. Only one of four prospective studies has shown that more frequent episodes of infection cause a more rapid decline in lung function [3]. Placebo-controlled studies involving small numbers of patients have provided conflicting evidence of the efficacy of antibiotics in acute exacerbations of COPD, but a recent meta-analysis concluded that overall there was a small benefit [4]. The study of ANTHONISEN et al. [5] is often cited when discussing antibiotics and COPD. In this study, 173 patients with chronic bronchitis were followed for 3.5 yrs during which time they had 362 exacerbations. Antibiotics or placebo were given in a randomized, double-blind, crossover fashion. Three levels of severity of exacerbation were recognized: the most severe comprised of worsening dyspnoea with increased sputum volume and purulence; a lesser grade was any two of these symptoms; and the least severe grade was any one of these symptoms with evidence of fever or an upper respiratory tract infection. Three antibiotics were used: amoxycillin, cotrimoxazole and doxycycline; the choice being made by the physician. There was a significant benefit from antibiotics which was largely accounted for by patients with the most severe exacerbations, whereas there was no significant difference between antibiotic and placebo in patients who had only one of the defined symptoms. A major criticism of this study was that no microbiology was performed, although this does reflect usual clinical practice. Most antibiotic trials have compared a new antibiotic with an established compound for the purpose of new product registration and licensing. Equivalence is the desired outcome of such trials, and as a consequence patients have been included with poorly defined disease of uncertain severity [6]. These trials have contributed very little to our knowledge about infection and COPD, and have failed to show that increased antibiotic potency in vitro translates into clinical superiority.

COPD encompasses several conditions (airflow obstruction, chronic bronchitis, emphysema) that often coexist,

Correspondence: R. Wilson, National Heart and Lung Institute, Emmanuel Kaye Building, Manresa Road, London SW3 6LR, UK. Fax: 441713518338. and the importance of bacterial infection may vary depending on the dominant pathology. For example, mucus hyper-secretion is particularly associated with mortality from an infectious cause [7]. Earlier studies used sputum culture to investigate the presence or absence of infection in COPD and determine the bacterial species involved. Most of the species isolated were nasopharyngeal commensals, and therefore, contamination may have occurred during expectoration. This risk can be reduced by using a Gramstain to demonstrate the presence of neutrophils which confirm infection and the absence of squamous cells which suggest an upper respiratory tract sample. Homogenization of the sputum sample and quantitative culture techniques can also be used to reduce sampling error and determine the level of infection. There is general agreement that the major pathogens isolated from sputum during acute exacerbation of COPD are nontypable Haemophilus influenzae, Streptococcus pneumoniae and Moraxella catarrhalis [2, $3]$, but several studies have shown that these species can be isolated from patients during stable periods, as well as during exacerbations [8-11]. One study did find an increase in the frequency with which bacteria were isolated from the same patients during exacerbations compared to stable periods [12], but this has not been a uniform finding.

The protected specimen brush (PSB) catheter, which is introduced during bronchoscopy, is used to sample lower respiratory tract secretions, and has much less risk of nasopharyngeal contamination since the distal catheter plug is only dislodged when the bronchoscope is in position to take the sample. The brush is retracted into the catheter before the whole unit is removed from the bronchoscope. The brush is aseptically cut into a vial containing Ringer's lactate solution or its equivalent, agitated to ensure all the bacteria are removed, and transported quickly to the laboratory where a quantitative culture is performed. The technique is well established in patients with pneumonia who have not received antibiotics $[13,14]$, but its application in COPD, where there is not an area of consolidation to direct the investigation, has been less well investigated. Monso et al. [15] studied 40 COPD patients during a stable phase of their illness, and found positive PSB cultures in onequarter. $H$. influenzae and $S$. pneumoniae were the predominant species detected which is in agreement with the results obtained in previous studies by sputum culture. Some of the same patients were studied again during a subsequent exacerbation. The number with positive PSB cultures increased to about $50 \%$ of the patients, and although the same species were predominant, the bacterial counts were higher. MARTINEZ et al. [16] studied patients with exacerbations requiring hospital admission who had not received antibiotics for 15 days. Fifteen of 20 patients 
(75\%) had positive PSB cultures, and $H$. influenzae and $S$. pneumoniae were isolated in greater numbers than other species that are usually regarded as nonpathogenic, e.g. $S$. viridans. FAGON et al. [17] studied patients with severe exacerbations of COPD which lead to hypercapneic respiratory failure, intubation and mechanical ventilation. Within $24 \mathrm{~h}$, prior to any antibiotics being given, a PSB sample was taken from a segmental bronchus with most secretions. There was bacterial infection in half of the cases, but the species detected were much more diverse, and $H$. parainfluenzae was the most frequent. With the exception of fever, which was significantly higher in those patients with a positive PSB culture, the clinical features of the exacerbation were similar in patients with and without infection; outcome measures were also similar in the two groups, although the majority of those patients with negative PSB cultures did receive empirical antibiotics.

Further studies using PSB are required to explore its use in COPD. Whether a certain predetermined site should be selected from which all biopsies should be taken during a study, or whether an inflamed bronchus or one with visible secretions should be chosen during the bronchoscopy to try to increase the yield has not been investigated. Although most studies have defined $10^{3}$ colony-forming units (cfu) $\cdot \mathrm{mL}^{-1}$ as a significant culture, this is a figure taken from pneumonia studies, and some workers in COPD have accepted lower numbers. There may be a large sampling error, and to date no studies have compared PSB cultures taken from different parts of the bronchial tree. In this issue of the Journal, Monso et al. [18] have investigated the repeatability of PSB taken from the same site during a single bronchoscopy. Overall repeatability was quite good, 13 of 18 patients had pairs of samples with bacterial growth $>$ or $<10^{3} \mathrm{cfu} \cdot \mathrm{mL}^{-1}$, but the agreement of positive cultures for individual bacterial species was much more variable (table 2 of the paper). Only five of 13 species were isolated with $\geq 10^{3} \mathrm{cfu} \cdot \mathrm{mL}^{-1}$ from both samples. One would expect this variability to be even greater if different sites had been chosen.

Monso et al. [18] in the study described above, and ZALACAIN et al. [19] in a study which is also published in this issue of the Journal, have both used PSB to investigate risk factors for bacterial colonization of the lower respiratory tract in stable COPD patients. Twenty-two per cent of patients in the former study and $40 \%$ in the latter had lower airway bacterial colonization (LABC). Nontypable $H$. influenzae was the most common isolate in both studies. The type of patient being studied has been well defined (stable chronic bronchitis without reversible airflow obstruction) and there were careful exclusion criteria in both studies. I think that this is a very important consideration in the design of future studies, since it is unlikely that LABC will be consistent across the COPD spectrum and careful definition of the type of patient being investigated will allow comparisons of results to be made between different studies. Both studies found that current cigarette smoking predisposed to LABC. Monso et al. [18] did not find an association between LABC and forced expiratory volume in one second (FEV1), whereas there was an association with more severe airflow obstruction (FEV $1<50 \%$ predicted) in the study performed by ZALACAIN et al. [19]. The latter study contained more patients with severe airflow obstruction, which may explain this difference.
The presence of LABC alone does not confirm the importance of bacterial infection in the pathogenesis of COPD or its exacerbations, since LABC could just be a marker of the severity of the airway disease. The study by FAGON et al. [17] described above, could be interpreted as showing that bacteria are passengers rather than active participants in the inflammatory process. LABC probably represents a balance in which the impaired host defences are able to limit the numbers of bacteria, but not eradicate them. Bacteria bind avidly to mucus, which suggests that infection may be more common in patients with chronic bronchitis, and bacteria produce toxins which in vitro damage epithelial cells and stimulate cytokine production $[3,20,21]$, so there is potential for LABC to be detrimental to the patient. A study presented at the recent European Respiratory Society meeting in Geneva showed that there were higher neutrophil counts, and elevated interleukin-8 (IL-8) and tumour necrosis factor (TNF) levels in bronchoalveolar lavage performed on stable chronic bronchitic patients with LABC compared to those with sterile PSB examinations [22]. Neutrophil counts were significantly inversely correlated with FEV1 in this study. Should this result be confirmed by other studies, and there are no confounding factors such as current cigarette smoking, it will provide us with very important evidence that $L A B C$ is a stimulus for airway inflammation.

Bacterial infection in acute exacerbations of COPD could be viewed as an upset in the balance between LABC and the host defences. This could occur for several reasons: a change in the bacterium colonizing the airway (e.g. acquisition of a new strain that is not recognized by the host defences or a change in the antigenic profile of the colonizing strain), or a reduction in the host defences (e.g. after a viral infection or due to air pollution). The earlier study by Monso et al. [15] has provided evidence that bacterial numbers increase during exacerbations, a finding which is important if they have a causal role. The rise in bacterial numbers would then provide a stimulus for increased inflammation, which in turn would lead to the symptoms of an exacerbation. However, another study using sputum culture found no relationship between bacterial numbers and sputum purulence [8], so further studies are required. About $40 \%$ of the patients with the most severe exacerbations in the ANTHONISEN et al. [5] study recovered whilst taking placebo, emphasizing that these are mucosal infections and the inflammatory response is quite often successful in overcoming the infection. Whether antibiotics can speed up recovery has received scant attention in clinical trials. A better understanding of risk factors for $\mathrm{LABC}$ would also be important since one could then identify patients prospectively who are more likely to get significant airway infections.

Monso et al. [18] and ZALACAIN et al. [19] both report isolation of bacterial species via PSB that are not usually regarded as respiratory pathogens, e.g. S. viridans. Our perception of what is a pathogenic species can change, for example $M$. catarrhalis was not regarded as a pathogen for many years. There is also little information about the propensity of different species to stimulate inflammation, and even different strains of the same species may vary in their ability to elicit an inflammatory response [23]. Consequently, the total bacterial load could be just as important as the species involved. In future studies it is probably best to follow the format adopted in these two papers, that 
is to record all isolates and analyse the results by total numbers of bacteria isolated and also by potential pathogenic bacteria.

Protected specimen brush is quite expensive and involves an invasive procedure, therefore it is likely that in chronic obstructive pulmonary disease it will remain a research tool. Future studies should seek to confirm the importance of lower airway bacterial colonization as an inflammatory stimulus, and seek to understand the dynamics of the interaction between the host and the bacterium during an exacerbation. Bacteria may be passive bystanders during an exacerbation or active participants in the inflammatory process. Finally, after 30 yrs of trying, we are beginning to answer this basic question.

\section{References}

1. Leading article. Antibiotics for exacerbations of chronic bronchitis. Lancet 1987; 11: 23-24.

2. Ball P, Tillotson G, Wilson R. Chemotherapy for chronic bronchitis controversies. La Presse Medicale 1995; 24: 189-194.

3. Murphy TF, Sethi S. Bacterial infection in chronic obstructive pulmonary disease. Am Rev Respir Dis 1992; 146: 1067-1083.

4. Saint S, Bent S, Vittinghoff E, et al. Antibiotics in chronic obstructive pulmonary disease exacerbations: a metaanalysis. J Am Med Assoc 1995; 273: 957-960.

5. Anthonisen NR, Manfreda J, Warren $\mathrm{CPW}$, et al. Antibiotic therapy in exacerbations of chronic obstructive pulmonary disease. Ann Intern Med 1987; 106: 196204.

6. Wilson R, Tillotson G, Ball P. Clinical studies in chronic bronchitis: a need for better definition and classification of severity. J Antimicrob Chemother 1996; 37: 205-208.

7. Prescott E, Lange P, Vestbo J. Chronic mucus hypersecretion in COPD and death from pulmonary infection. Eur Respir J 1995; 8: 1333-1338.

8. Storey PB, Morgan WKC, Diaz AJ, et al. Chronic obstructive airway disease. Bacterial and cellular content of the sputum. Am Rev Respir Dis 1964; 90: 730-735.

9. Calder MA, Schonell ME. Pneumococcal typing and the problem of endogenous or exogenous re-infection in chronic bronchitis. Lancet 1971; i: 1156-1159.

10. McHardy VU, Inglis JM, Calder MA, et al. A study of infective and other factors in exacerbations of chronic bronchitis. Br J Dis Chest 1980; 74: 228-307.

11. Groeneveld K, Alphen LV, Eijk PP, et al. Endogenous and exogenous reinfections by Haemophilus influenzae in patients with chronic obstructive pulmonary disease: the effect of antibiotic treatment on persistence. $J$ Infect Dis 1990; 161: 512-517.

12. Fisher M, Akhtar AJ, Calder MA, et al. Pilot study of factors associated with exacerbations in chronic bronchitis. Br Med J 1969; 4: 187-192.

13. Wimberley N, Faling J, Bartlett JG. A fibreoptic bronchoscopy technique to obtain uncontaminated lower airway secretions for bacterial culture. Am Rev Respir Dis 1979; 119: 337-343.

14. Chastre J, Fagon JY, Barnet-Lecso M, et al. Evaluation of bronchoscopic techniques for the diagnosis of nosocomial pneumonia. Am J Respir Crit Care Med 1995; 152: 231240.

15. Monso E, Ruiz J, Rosell A, et al. Bacterial infection in chronic obstructive airways disease: a study of stable and exacerbated outpatients using the protected specimen brush. Am J Respir Crit Care Med 1995; 152: 1316-1320.

16. Martinez JA, Rodriguez E, Bashda T, Buges J, Torres M. Quantitative study of the bacterial flora in acute exacerbations of chronic bronchitis. Chest 1994; 105: 976.

17. Fagon JY, Chastre J, Trouillet JL, et al. Characterization of distal bronchial microflora during acute exacerbation of chronic bronchitis; use of the protected specimen brush technique in 54 mechanically ventilated patients. Am Rev Respir Dis 1990; 142: 1004-1008.

18. Monso E, Rosell AT, Boret G, et al. Risk factors of lower airway bacterial colonization in chronic bronchitis. Eur Respir J 1999; 13: 338-342.

19. Zalacain R, Sobradillo V, Amilibia J, et al. Predisposing factors to bacterial colonization in chronic obstructive pulmonary disease. Eur Respir J 1999; 13: 343-348.

20. Wilson R, Dowling RB, Jackson AD. The biology of bacterial colonization and invasion of the respiratory mucosa. Eur Respir J 1996; 9: 1523-1530.

21. Khair OA, Devalia JL, Abdelaziz MM, Sapsford RJ, Davies RJ. Effect of erythromycin on Haemophilus influenzae endotoxin-induced release of IL-6, IL-8 and SICAM-1 by cultured human bronchial epithelial cells. Eur Respir J 1995; 8: 1451-1457.

22. Soler N, Ewig S, Agusti C, et al. Bacterial colonization and inflammatory response in patients with stable chronic obstructive pulmonary disease. Eur Respir $J$ 1998; 12: Suppl. 28, 230s.

23. Bresser P, van Alphen L, Habets FJM. Persisting Haemophilus influenzae strains induce lower levels of interleukin-6 and interleukin-8 in H292 lung epithelial cells than non-persisting strains. Eur Respir J 1997; 10: 23192326. 